\title{
DOES OCCUPATIONAL EXPOSURE TO ARGON LASER RADIATION DECREASE COLOUR CONTRAST SENSITIVITY IN UK OPHTHALMOLOGISTS?
}

\author{
G. B. ARDEN and M. J. HALL \\ London
}

\begin{abstract}
SUMMARY
The objects of the study were to determine: (1) whether United Kingdom ophthalmologists who used argon lasers had the elevation of colour-contrast thresholds previously discovered and (2) whether other users of argon lasers showed any unusual loss of colour vision. A total of $1072 \mathrm{UK}$ ophthalmologists filled in a questionnaire about their professional use of lasers, the length of time spent operating, and their out-ofdoors activities. Their colour vision was then tested by a new sensitive system, and if any abnormality was detected, a clinical eye examination was performed. The results were as follows: (1) Colour vision testing was shown to be reliable. Any self-selection bias was excluded. Test-retest variability was small. Normal results did not change during the survey. (2) A number of men with high red-green thresholds were discovered. Some were aware of their congenital insensitivity. The frequency of all such defects was less than the known incidence of congenital colour deficiency in the male population. (3) Additionally a number of high tritan (blue-yellow) thresholds were encountered, some associated with reported diabetes and hypertension. In other cases of this type, undetected or unacknowledged systemic disease may be present. (4) After making allowance for all these incidental causes of loss of colour vision, and for the effect of age on colour vision (which is very small) only four of the sample were >2 SD above normal. (5) However, the average blue-yellow thresholds of ophthalmologists were slightly and highly significantly raised compared with normal, in the first year of the survey. During the second and third years, the mean thresholds declined to normal. Similar but less significant findings were found for protan thresholds. It is concluded that the enhanced safety precautions recently introduced are associated with a recovery of colour vision in this population,
\end{abstract}

Correspondence to: G. B. Arden, Applied Vision Research Unit, City University, London EC1V 7DD, UK. demonstrating that any changes to individuals were reversible. Colour vision screening has proved able to detect mild ocular abnormalities due to systemic and congenital disease.

In the past 20 years the use of lasers in ophthalmology has become routine, especially in the treatment of diabetic eye disease, ${ }^{1}$ where the argon laser is used in pan-retinal photocoagulation. To achieve sufficient power, many original lasers employed the wavelengths $488 \mathrm{~nm}$ (blue) and $514 \mathrm{~nm}$ (green). A low power was used to aim the beam at a desired spot on the retina, which, however, was in a range that the ANSI standard considered might approach the hazardous if viewed directly. ${ }^{2-4}$ Although no acute retinal damage was observed in safety trials, it has been shown that with argon lasers employing a blue aiming beam, acute loss of tritan colour contrast sensitivity occurs in the user. ${ }^{5}$ These changes in the surgeon's vision were detected soon after finishing a treatment session, but in a small sample it was found that chronic elevation of threshold could occur. ${ }^{6,7}$ The acute change in sensation was apparently due to the intensity of the aiming beam. When it was employed at low or medium settings, no blue threshold elevation occurred, and no elevation was seen if the surgeons used dye lasers which emit longer, less hazardous wavelengths. ${ }^{7}$ An explanation of the result was that the aiming beam, reflected from optical surfaces (mainly the lenses used for viewing the retina) back into the operators' eyes ('flashbacks'), cumulatively affected vision.

To investigate this possibility 211 German ophthalmologists at the 1990 Annual Meeting of the Deutches Ophthalmologische Gesellschaft were tested. ${ }^{8}$ The subjects were divided into two roughly equal groups, which differed only in the fact that one employed lasers (and operated under bright lights) 
while the others did not. Both groups used other ophthalmological tools, and were from similar educational, social and economic groups. The nonlaser users therefore formed a useful control. About one-third of the laser users had tritan colour contrast thresholds elevated $>2$ standard deviations above the mean of the non-laser users. However, for protan colour contrast the results were within the normal range. There was a significant correlation between duration of exposure to argon blue and the degree of elevation of tritan threshold. There appeared to be a small, and only possibly significant correlation between elevation of the protan threshold and exposure to operating theatre conditions, which seemed to be synergistic with losses associated with argon lasers. The threshold elevations were lower than found in congenital colour deficiencies; however, the survey covered a sample of $<20 \%$ of all German ophthalmologists, so that the subjects tested were not necessarily representative.

While loss of blue vision is not of great importance, the possibility that even minor elevation of red-green thresholds occurred was worrying, since such damage could accumulate over a working lifetime and might contribute to ageing changes. For these reasons a larger survey of United Kingdom ophthalmologists was undertaken and this paper reports the results obtained. Before the study began, the makers of ophthalmic lasers introduced new models in which the aiming beam was red or green and a number of older lasers, which used both argon blue and argon green, were modified with blueabsorbing filters in the viewing oculars, or the green wavelengths only were employed. These measures considerably reduced the numbers of ophthalmologists routinely exposed to bright blue 'flashbacks' from argon lasers, so the study reported on below was carried out under quite different conditions to those prevailing in the initial reports.

The general findings are that compared with the earlier survey, changes in colour contrast threshold are much reduced, and in the last 3 years a significant improvement in threshold has occurred in the tested population. Thus it appears that the measures to reduce exposure have been successful, and the chronic threshold elevations previously noted are slowly reversible.

\section{METHODOLOGY}

Before every test, each subject was asked to fill in a confidential questionnaire to discover the exposure to laser light the subject had experienced, and whether there was any other possible cause for an abnormality in colour vision.

\section{Equipment for Colour Vision Testing}

The stimuli for colour vision testing were displayed on a BARCO Industries CCID 7551 colour monitor with RGB inputs, for which the chromaticity of the phosphors is known to three decimal places. The contrast and luminance of the images on the monitor are controlled by the software (described below). The equipment was recalibrated before each use. The images used to determine colour contrast threshold were constructed by the program and consisted of alphabetic letters (a subset of the Sloan optotypes) which were displayed transiently on the monitor screen in varying colour contrast to the background. The luminance of the colour and the background were always the same. Colour contrast was varied along colour confusion axes for tritanopic and protanopic observers chosen to be orthogonal in colour space. The letters subtended 2.5 degrees at the eye, i.e. were in terms of test types equivalent to $2 / 400$, so that colour thresholds, not acuity thresholds, were tested. A refinement possible with this system is the production of an automated sequence which conducted the entire test without any input from the tester, excepting a signal that he was satisfied that a stable threshold had been achieved.

\section{Persons Tested}

All United Kingdom (England, Scotland, Wales and Northern Ireland) ophthalmologists were eligible to participate in the study. A list of ophthalmologists in post in the grades Consultant, Senior Registrar, Registrar, Senior House Officer, Associate Specialist, Clinical Assistant and Staff grade was compiled from returns made by each of the 232 District Health Authorities in the United Kingdom. The initial list was compiled during the period May-August 1991, and updated each subsequent January. Those whose names did not appear on subsequent listings from the District Health Authorities were traced via any entries in The Medical Directory (1992) and via Medical Staffing departments at their last known place of work. An initial list of 1587 individuals.was created, expanding to 2204 after the final update in 1993. A summary of findings is given in Table I. The losses of subjects over the 3 years include both immigration and emigration and some subsequent return.

Table I. Subjects of main enquiry 


\section{Organisation and Administration of Colour Vision Testing}

Sixty-nine hospitals were selected as test centres at which to base the study. These provided facilities (test rooms and waiting areas) for between 2 hours and 3 days. Each ophthalmologist in the 38 Regional Health Authorities of England (14), Wales (9) and Scotland (15) working in the vicinity of a test centre was sent a personally addressed letter $2-3$ weeks before the screening date(s) inviting him or her to participate. This was followed by a reminder letter 1 week prior to testing. Tests were also carried out at major UK ophthalmic conferences: the Oxford Ophthalmological Congress (1991, 1992, 1993), the College of Ophthalmologists' sixth and seventh annual meetings $(1992,1993)$ and the Cambridge Symposium (1991). Appointments were made via the secretary of the senior Consultant at the test hospital, though subjects could present themselves at the test room without previously booking an appointment.

The test was voluntary, as was made clear in the invitation letter sent to each ophthalmologist, and again in the reminder letter. In addition, we made it clear that the test results would be treated in confidence. All participants were informed of their test results, and whether they were unusual. In cases (see below) in which abnormality was detected, we felt that the subject should be advised to have a clinical examination. In such cases, a further letter was sent to the person involved, and he was asked if he preferred to be examined at Moorfields Hospital, or at his local centre. The latter was preferred in many cases and, if so, we requested permission to obtain the results of such examinations, which was invariably granted.

No one who wished to participate was denied the opportunity to do so, although a number of exclusion criteria have been introduced for the final analysis.

\section{Limitations and Exclusions}

Data from all subjects with known congenital colour defects were analysed separately, as were data from those participants self-reporting diabetes, hypertension, ocular disease and individuals taking medication with known or possible effects on colour contrast thresholds. Those who provided insufficient information about laser use or operating microscope use were also removed from the main data base.

\section{Testing on Repeated Occasions}

One of the major incentives which led ophthalmologists to submit to our tests was their knowledge of our previous results which showed threshold elevations in about one-third of cases. ${ }^{6-8}$ However, after the first test they knew whether their own thresholds were elevated or not. In addition they were well aware of the changes in laser safety which had recently occurred. Most were working with lasers which transmitted argon green only, or in which blue-absorbing filters had been used to protect the viewers' eyes from the aiming beam, or in which the aiming beam was helium-neon. For this reason the incentive to repeat the test after 1 or more years was much reduced, and this probably accounts for the fact that in the later years of the study we were able to attract a smaller number of persons for repeat tests, despite our best efforts.

\section{Colour Vision Test Routine}

Only the dominant eye of each subject was tested routinely. The test room was lit only by artificial light, turned off during the test so general illumination was reduced to $<1 \mathrm{~cd} / \mathrm{m}^{2}$, i.e. less than $3 \%$ of the screen illumination; reflections on the screen from objects in the test room thus did not influence the results.

Each subject was first shown a screen on which a red square changed to a green square, and reversed back to red at $22 \mathrm{~Hz}$, with a $50 \%$ red/green duty cycle. The screen appeared to contain a yellowish flickering square. By pressing one of the buttons on a 'mouse' the luminosity of the green could be reduced. The colour changed slightly - became more yellow - and the flickering sensation diminished. A point was reached at which the flicker sensation vanished. If the green luminance was further reduced, the flicker reappeared as the colour changed to orange and finally to a red. The second mouse button, when pressed, increased the luminosity of the green. Subjects set the screen by pressing the appropriate mouse button until the screen display stopped flickering. The operator then ended the period of observation by a keyboard entry on the computer. The subjects were next shown a rapidly flickering green/blue square, and reduced the green luminance until flicker ceased. This entire sequence was repeated until the standard deviation of the R:G luminance setting was $3 \%$ of the mean, or less.

The screen luminance was calibrated in candelas per metre squared, a measure derived from the CIE standard observer (1932), so the mean R:G ratio for normal subjects was 1.00. A gross abnormality in the setting indicated anomalous trichromacy or dichromacy, and this test is similar to the use of an anomaloscope. Small deviations from the standard occur in normal observers, and reflect differences in the coloration of the lens, or the density of the yellow macular pigment. Thus, the relative perceived luminance of the phosphors varies from person to person. The settings were used by the program to fine tune the subsequent colour vision test so all colours were equiluminous for each individual. 
Table II. Correlation coefficients between different questionnaires

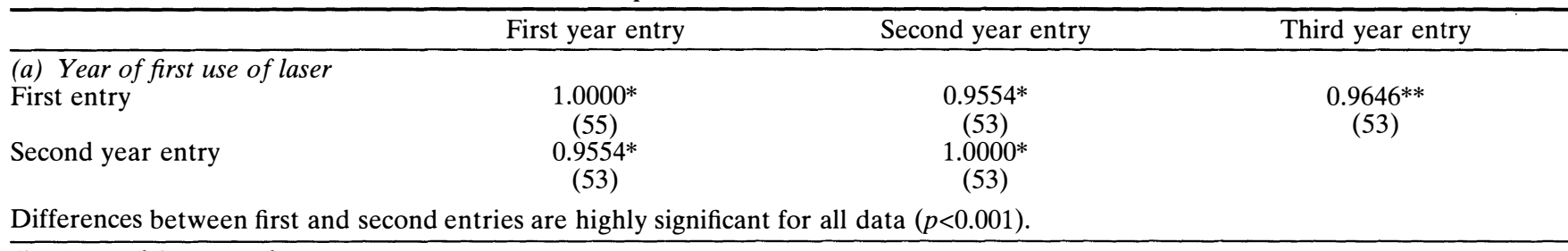

(b) Year of first use of operating microscope

First year

$\begin{array}{cc}1.0000^{*} & 0.9341^{*} \\ (50) & (47) \\ 0.9341^{*} & 1.0000^{*} \\ (47) & (55)\end{array}$

$0.9379 * *$

Second year

$1.0000^{*}$

Differences between first and second entries are highly significant for all data $(p<0.001)$.

(c) Number of hours of laser use

First year entry

Second year entry
$1.0000^{*}$
$(422)$

$0.7513^{*}$

(422)
$0.7513^{*}$

$(422)$

1.0000*

$(422)$

Differences between first and second entries are highly significant for all data $(p<0.001)$.

(d) Hours spent operating

$\begin{array}{lcc} & 1.0000^{*} & 0.7756^{*} \\ \text { First year entry } & (550) & (549) \\ \text { Second year entry } & 0.7756^{*} & 1.0000^{*} \\ & (549) & (549)\end{array}$

Differences between first and second entries are highly significant for all data $(p<0.001)$.

The numbers in the samples are given in parentheses.

*Pearson correlation coefficient; **Spearman correlation.

\section{Measurement of Colour-Contrast Thresholds}

The subjects covered one eye and a flashing coloured letter appeared on the monitor screen for $200 \mathrm{~ms}$ every second. The subject was asked to identify the letter. If this could not be done, the colour contrast increased: if the letter was successfully identified, the colour contrast was decreased. A new image (another letter) was presented to the subject. The change in colour contrast was determined by a modified binary search routine. ${ }^{9}$ Only the protan and tritan colour axes were tested. In the previous trials the deuteran axis was also tested, but no further information regarding either blue light damage or congenital colour deficiency was provided by these measurements and therefore blue-green (deuteran) contrast sensitivity was routinely tested in this survey.

Table III. Similarity of $65 \%$ of population tested to sub-sample with $>90 \%$ tested (population numbers 1027, sub-sample contains 45)

\begin{tabular}{lcc}
\hline & Mean + SD & SEM \\
\hline Protan & $6.826+7.144$ & $1.065^{*}$ \\
Sub-sample & $6.615+5.686$ & $0.177^{*}$ \\
Population & & \\
Tritan & $12.608+4.232$ & $0.631 \dagger$ \\
Sub-sample & $12.749+6.129$ & $0.191 \dagger$ \\
Population & & \\
\hline
\end{tabular}

*Levene's test for equality of variance: $F=0.518, p=0.472$.

Variances equal: $t=0.24$, two-tail significance 0.810 .

Variances unequal: $t=0.20$, two-tail significance 0.846 .

†Levene's test for equality of variance: $F=0.179, p=0.672$.

Variances equal: $t=-0.15$, two-tail significance 0.879 .

Variances unequal: $t=-0.21$, two-tail significance 0.832 .

\section{RESULTS: SUBSIDIARY PROBLEMS}

\section{Validation of Questionnaire Data}

The questionnaire was designed to provide information about the ophthalmologist's exposure to laser light and about his or her general health and eye condition. No validation could be performed about the medical conditions which ophthalmologists admitted to. It is possible, therefore, that the incidence of hypertension, diabetes and general disease was under-reported, but this is not considered as serious; as can be seen below, the conclusions of the analyses are not changed if it is considered that a number of anomalously high tritan thresholds were due to systemic disease.

Exposure to laser light was estimated by the ophthalmologists, and there was again no reason to suppose that they were biased. However, because the duration of exposure is one of the main variables in the study, validation was considered necessary. External validation, by direct comparison between the questionnaires and the laser log books from within a case hospital was not possible because of deficiencies in the records of the largest centres where lasers were used. Laser log books suffer from incomplete data: not all patients are logged in the book, and the number of burns is not recorded.* An

*If the laser log book were to contain the following information validation would be possible: (1) name of laser operator, legibly written, and not just initials or the name of the consultant in charge; (2) a measure of the duration of the procedure (for example, number of burns, wattage, duration and size). It would be helpful if such a system could be computerised and linked to a lock on the laser console. 
Table IV. Test-retest changes

\begin{tabular}{|c|c|c|c|c|c|c|}
\hline & \multicolumn{2}{|c|}{ First visit (V1) } & \multicolumn{2}{|c|}{ Second visit (V2) } & \multicolumn{2}{|c|}{ Ratio V1:V2 } \\
\hline & Protan & Tritan & Protan & Tritan & Protan & Tritan \\
\hline$n$ & $8^{\mathrm{a}}$ & $9^{a}$ & 7 & 8 & 7 & 8 \\
\hline Mean & 5.17 & 9.68 & 5.49 & 8.66 & 0.96 & 1.10 \\
\hline SE & 0.41 & 0.80 & 0.61 & 0.67 & 0.10 & 0.07 \\
\hline
\end{tabular}

${ }^{a}$ One person protanomalous: one did not return for second trial.

There were no significant changes on retesting (sign test, two-tailed: $p=0.289$ ).

alternative (internal validation) is to compare the several questionnaires filled out by the same individual to see whether there were any differences in recall on various occasions. If the subjects filled questionnaires entirely differently on each occasion, we could not trust their recollection of the use of lasers/operating microscopes.

Results are shown in Table II. The recall for the year when lasers or operating microscopes were first used was excellent (Table II $a, b$ ). This part of the survey was carried out on a subgroup who had filled in the questionnaire three times, and refers to the first and third entries. An appropriate test is Spearman's correlation coefficient, as Pearson's correlation coefficient assumes normal distribution of data, a condition which is not met. Both correlation coefficients are presented to show that the differences between the two are not great. Perfect correlation of course exists between year 1 and year 1 results, but the correlation coefficients are lower when a year has intervened between the fillingin of the two questionnaires. Memory and 'telescoping' errors are present - failure to remember an event and the bringing forward in time of an event that occurred earlier than the subject recollects. The correlations for the operating microscope data are remarkably similar to those of the argon laser data. The wording of the questionnaire was slightly altered between years 1 and 2 of the screening, and this may account for some of the reduction from unity of the correlation coefficients obtained. The hourly use of lasers and microscopes (Table II $c, d$ ) involved remembering the number of sessions per week, often for a number of years. Table II $c$ and $d$ are from a larger group which filled in these items on two occasions (most commonly, the years 1991 and 1992). As can be seen the correlations are not quite so high but the data can be used to interpret changes in vision (if found) with usage of lasers: perfect recall of past events when questions are asked on two occasions a year apart is not possible.

\section{Possible Selection Bias}

One of the problems in the initial (German) study was that a minority of ophthalmologists were tested, and therefore it is possible that those with poor vision formed a disproportionate number of our sample. In this investigation, despite all the publicity, only about $65 \%$ of ophthalmologists at most sites were tested. It was therefore necessary to determine whether these represented the entire population. In four sites, we returned to test subjects until more than $90 \%$ of the doctors had been investigated. Thus an analysis for non-response bias could be undertaken.

Table III shows that the colour-contrast thresholds for the subgroup in which almost all ophthalmologists were tested were essentially identical to those of the population tested, which excluded those who found it 'difficult' to be tested. Therefore we can conclude that had it been possible to test every ophthalmologist in the country, the results obtained would have been similar to those achieved, with a high level of probability. An alternative statistical method was also employed: the non-parametric Mann-Whitney $U$-test. This gave similar results to those of Table III: for protan, $Z$ (corrected for ties) $=-1.001$, two-tailed $p=0.3167$; and for tritan, $Z$

Table V. Long-term stability of normal thresholds

\begin{tabular}{lccc}
\hline Date: & 10 March 92 & 3 July 92 & 2 August 93 \\
Group name: & NPL & MEH & 47 \\
Mean age (years): & 36 & 31 & 7 \\
$n:$ & 14 & 23 & 5.27 \\
Mean protan: & 5.57 & 5.43 & 0.84 \\
SE: & 0.34 & 0.33 & 11.72 \\
Mean tritan: & 12.14 & 10.92 & 1.11 \\
SE: & 0.57 & 0.40 & Tritan \\
& Protan & $11.43^{\mathrm{c}}$ & 0.33 \\
Averaged age (years) & 36 & 11.43 & 0.32 \\
Grand mean & $5.51^{\mathrm{a}}$ & 0.18 & \\
SE & 5.51 & 0.17 & \\
Grand mean & & & \\
SE & &
\end{tabular}

${ }^{\mathrm{a} E x c l u d i n g}$ congenital colour defectives.

'Excluding 2 'outliers'.

'Age adjusted to mean age of ophthalmologists. 
Table VI. Thresholds prior to exclusions

\begin{tabular}{lrrrr}
\hline & Number & Mean & SD & SEM \\
\hline Protan & & & & \\
First test & 1072 & 6.624 & 5.751 & 0.176 \\
Second test & 406 & 6.340 & 5.332 & 0.265 \\
Third test & 143 & 6.300 & 5.358 & 0.448 \\
Tritan & & & & \\
First test & 1072 & 12.74 & 6.060 & 0.185 \\
Second test & 406 & 12.28 & 4.480 & 0.222 \\
Third test & 143 & 11.91 & 4.997 & 0.416 \\
\hline
\end{tabular}

(corrected for ties) $=-0.647$, two-tailed $p=0.5174$. This method of analysis likewise supports the assertion that there is no significant difference between the major sample and the sub-sample.

\section{Test-Retest Variability}

A separate formal test of test-retest variability was not done, because the interval between testing the main group was approximately 1 year. In previous work,,$^{10}$ no difference was found between results of first and second tests when the interval was shorter than this. One of the subgroups studied (Loughborough University) was re-visited after 6 weeks, and the same personnel tested. They had been using lasers, but the first test was done after a week in which lasers were not used, and these results were all normal (Table IV). There was no difference 6 weeks later.

\section{Stability of Thresholds}

Three normal groups were tested during the course of the investigation: The results are given in Table V. It can be seen that the equipment used provided similar results for normals from January 1992 to July 1993. Note no adjustment has been made for age in the upper entry of the table, while at the foot the averages of all the results are shown after a correction for age, so these results can be used as a normal comparison for results obtained on the ophthalmologists.

\section{MAIN RESULTS}

The 1622 tests considered were performed between June 1991 and April 1994. The crude results are: (mean values are followed by the standard deviation of the mean). The protan threshold was $6.52 \pm 5.61$ and the tritan threshold was $12.55 \pm 5.62$. These figures include all subsequent exclusions. Before any inferences can be drawn from such figures, considerable analysis is required. This is described in the following pages.* Only then can questions be answered about the influence on colour vision of

Table VII. Colour vision thresholds of known congenital colour defectives $(n=35)$

\begin{tabular}{lcc}
\hline & Mean & SD \\
\hline Protan threshold & 30.10 & \pm 17.23 \\
Tritan threshold & 15.05 & \pm 8.57 \\
\hline
\end{tabular}

Table VIII. Colour vision thresholds of persons known to be diabetic $(n=15)$ or suffering from systemic hypertension $(n=26)$

\begin{tabular}{lrc}
\hline & Mean & SD \\
\hline Diabetics & 7.67 & \pm 4.36 \\
Protan threshold & 21.47 & \pm 14.53 \\
Tritan threshold & & \\
Hypertensives & 7.68 & \pm 6.78 \\
Protan threshold & 15.82 & \pm 5.50 \\
Tritan threshold &
\end{tabular}

duration of exposure and amount of exposure to laser light. Thus, the mean thresholds of ophthalmologists are greater than for the normals, but part of this may be simply explained: subjects with abnormal colour vision - from any cause - returned for testing every year. Because only one subject was tritanomalous, tritan test results are scarcely influenced by congenital colour defects. However, the uncorrected tritan results include persons with acquired disease. Table VI shows the crude results, before excluding any data, separating subjects into those from whom one or more tests were obtained. The table shows that there appears to be a slight decrease in thresholds in the later years of the study, but the data require correction before any inference can be drawn.

\section{Data Excluded from the Main Analysis}

Congenital Colour Defectives. A number of colour defectives identified themselves as such (Table VII). All were male: Only two tritanomalous subjects were encountered, corresponding to the population incidence of 1 in 2000 . The total number of protan and deutan anomalous trichromats and dichromats in the male population is 1 in 8 ; in the female population it is approximately 1 in 250 . The number of men in our sample is 810 , and of women, 362 . Thus we would expect, on a random basis, that 101 men would be affected, but as indicated only ${ }^{\circ} 35$ identified themselves. Of course it is possible that there is a tendency for persons with abnormal colour vision not to take up surgery, but it is equally possible that among our ophthalmologists are a number with mild colour vision defects who are unaware of their problems. These thresholds could greatly influence our results, so analyses should be carried out on the basis of both possibilities.

Acquired Colour Deficiency. Diabetic retinopathy is known to cause loss of colour vision. Furthermore,

*It may assist the reader to give an outline of the method of analysis. First, data from subjects with obvious disabilities must be removed: for example, subjects with systemic diseases. Now, for protan vision, all colour defectives should be removed. However, even after this is done, some very high protan results remain. But the reported incidence of congenital colour defects in the subjects is much less than in normal populations, so it is reasonable to suppose that these values belong to persons who forgot to report (or did not know) their disability. The same may apply to high tritan values: subjects may not admit to, or know of a disease condition: so the results should be analysed with and without these high values. Next, the effect of age must be factored out. 
Table IX. Thresholds after exclusions

\begin{tabular}{lccccc}
\hline & No. of tests & Mean & SD & SEM & No. abnormal $^{\mathrm{a}}$ \\
\hline Protan & & & & & \\
First test & 958 & 5.804 & 2.310 & 0.075 & 19 \\
Second test & 345 & 5.529 & 1.767 & 0.093 & 8 \\
Third test & 125 & 5.309 & 1.189 & 0.106 & \\
Tritan & & & & & \\
First test & 958 & 12.37 & 5.306 & 0.171 & 29 \\
Second test & 345 & 12.02 & 4.093 & 0.215 & \\
Third test & 125 & 11.58 & 4.191 & 0.375 & 3 \\
\hline
\end{tabular}

${ }^{\mathrm{a}}$ Defined as having thresholds $>2$ SD above the mean.

loss of tritan sensitivity occurs in diabetics before there are obvious changes in the retina. We therefore included a question about diabetes in our questionnaire. Table VIII shows the results. Fifteen of the sample declared they had diabetes. In all cases their visual acuity was normal, and there were no known defects of vision. In all cases with raised tritan values, an eye examination was conducted, and no diabetic retinopathy was observed. Nevertheless, the average tritan sensitivity is considerably decreased. There are several previous reports of the raised thresholds of diabetics before the development of sight-threatening retinopathy. ${ }^{5,11,13-16}$ Our investigation confirms such findings. Note that there is no significant increase in protan threshold. Only 2 of these subjects reported that they were taking insulin; 5 were taking glibenclamide. There was no significant difference between the results of the insulin-dependent and non-insulin-dependent groups. Thus, type 1 and 2 diabetics may be equally impaired (a result also indicated by other studies: Arden, unpublished data). Note that it is possible that a number of other persons tested were diabetic but did not choose to make this fact known to us.

There have been no previous indications that controlled hypertensives without retinopathy have altered colour vision. Nevertheless, as Table VIII indicates, the average tritan colour vision sensitivity is considerably decreased. Although the protan thresholds are not increased to the same extent, they are higher than for the main group after excluding persons with known ocular and systemic conditions. The significance of this interesting finding should be the subject of further investigation. It is known that other indications of eye disease (for example, the macular drusen that precede agerelated maculopathy, or ocular hypertension as a precursor to glaucoma) are associated with a selective increase in tritan thresholds. ${ }^{10-12,17}$ We had no way of determining whether the other persons tested were normotensive, or whether colour vision changes were associated with any specific medication. Because of this finding, we have excluded this group from subsequent analyses.

Other Exclusions. Data were missing in 14 subjects; 12 subjects had acquired eye disease and 27 subjects, mostly diabetics and hypertensives, were taking medication. Results after all the exclusions mentioned above are shown in Tables IX-XIII.

\section{Corrected Thresholds}

It can be seen that after the exclusions, the protan mean thresholds decrease significantly, probably reflecting the proportion of red-green congenital colour anomalies in the male population. The decrease in tritan thresholds is less marked. The standard deviations decrease considerably for both tritan and protan tests, showing that much of the variation within the groups was due to the results excluded. Therefore despite the exclusions a number of results lie more than 2 standard deviations above the group mean. A decision must be taken about the significance of these 'abnormal' results.

\section{Effect of Outliers}

We considered this problem for the first test on each subject. In addition to the known colour defectives, some 19 persons had protan colour thresholds more than 2 SDs above the normal, without any other clinical signs. It is probable that these persons had mild colour anomalies, since then the proportion (54 in 810) rises towards the established incidence in the general population.* It seems likely that persons with colour vision problems are less likely to become ophthalmic surgeons, and this would explain why, with a relatively large sample, hereditary protan defects are under-represented.

We know from our previous work that protan thresholds are scarcely affected by the use of lasers, even when tritan thresholds are elevated, and in this investigation, as indicated, even tritan results are nearer the normal than we found previously. Therefore, there is no reason to suppose that persons with

\footnotetext{
*The point in colour space from which colour contrast was varied was not identical for tests of contrast sensitivity on protan and tritan colour confusion lines and varied slightly with the degree of severity of the defect, since only mild anomalies do not have changes in relative spectral sensitivity. The defect is best describel by a change in the major and minor axes of the MacAdam ellipse, which defines the area in colour space in which confusions occur. The angles of the major axes for protan and deutan defects are not identical, so that in this investigation, when only one confusion line was tested, protan and deutan defects are not distinguished, and the extent of the abnormality of the deutah defect would be reduced.
} 
elevated protan thresholds had acquired disease, and subsequent analyses excluded all persons with loss of protan sensitivity, presuming them to be congenital colour defectives.

In addition to the protan outliers, some 29 persons had loss of tritan sensitivity (values $>2$ SDs above the normal). The reasons for this are unknown but a simple answer might be that this was related to the use of lasers, or to the under-reporting of systemic disease. We have therefore analysed results both including and excluding these 19 tritan outliers.

\section{The Effect of Age}

Colour-contrast thresholds vary with age, but the change is small for tests of hue discrimination ${ }^{10-12,17,18}$ compared with the scores for tests involving memory and intelligence (e.g. the 100 hue test). In the present data we find a crude relationship as follows.

Before removing outliers:

Protan threshold $=4.32+($ age - mean age $)-$ 0.03807

Tritan threshold $=6.36+($ age - mean age $)-0.154$

After removing outliers:

Protan threshold $=4.352+($ age - mean age $) \times$ 0.039781

Tritan threshold $=6.875+($ age - mean age $) \times$ 0.1437

Because it is possible that exposure to environmental factors (such as excess light experienced through the use of YAG lasers, operating microscopes, argon lasers, and recreational UV exposure during sailing or skiing) increases with age, we also determined the relationship after using the program

Table X. Corrected changes in population colour vision as function of year of survey

\begin{tabular}{lccc}
\hline Protan colours & & & \\
Year: & $91-92$ & $92-93$ & $93-94$ \\
Mean: & $5.8694^{\mathrm{a}, \mathrm{c}}$ & $5.6538^{\mathrm{a}, \mathrm{b}}$ & $5.3896^{\mathrm{b}, \mathrm{c}}$ \\
$n:$ & 673 & 434 & 338 \\
SD: & 2.348 & 1.923 & 1.798 \\
SEM: & 0.091 & 0.092 & 0.098 \\
Tritan colours & & & \\
Year: & $91-92$ & $92-93$ & $93-94$ \\
Mean: & $12.815^{\mathrm{d}, \mathrm{e}}$ & $12.104^{\mathrm{d}, \mathrm{f}}$ & $11.184^{\mathrm{e}, \mathrm{f}}$ \\
$n:$ & 673 & 434 & 338 \\
SD: & 5.929 & 4.119 & 3.307 \\
SEM & 0.229 & 0.198 & 0.180 \\
\hline
\end{tabular}

${ }^{a}$ Levene's test for equality of variances: $F=8.794, p=0.003$. $t$-test for equality of means: $t=1.67, p=0.096$.

${ }^{\mathrm{b}}$ Levene's test for equality of variances: $F=15.784, p=0.000$. $t$-test for equality of means: $t=3.60, p<0005$.

${ }^{c}$ Levene's test for equality of variances: $F=1.643, p=0.200$. $t$-test for equality of means: $t=1.96, p=0.05$.

${ }^{\mathrm{d}}$ Levene's test for equality of variances: $F=15.974, p<0.0005$.

$t$-test for equality of means: $t=1.67, p=0.096$.

${ }^{\mathrm{e}}$ Levene's test for equality of variances: $F=30.281, p<0.0005$.

$t$-test for equality of means: $t=5.61, p<0.0005$.

${ }^{\mathrm{f}}$ Levene's test for equality of variances: $F=5.023, p=0.025$. $t$-test for equality of means: $t=3.44, p=0.01$.
'SPSS, Windows' to factor out any variability due to such causes. Results obtained in the first, second and third years of the investigation were treated separately. The influence of the variables of threshold was exceedingly small. Thus the influence of age on any change in threshold with time is positive (thresholds rise with age) and insignificant over the 3 year time span of this survey.

With the adjustment of thresholds to remove any effect of age, it is possible to determine whether the thresholds changed during the course of the survey (Tables X-XIII). To determine whether these changes were significant $t$-tests were used. (Note that in previous data, the first, second and third tests did not correspond precisely to the years of the survey.) These results indicate that in the population tested, after all the exclusions we could make, and after correcting for the effects of age, sensitivity improved during the survey period: however, the increase in tritan sensitivity was much greater and the significance of the result is higher than for the protan readings. Table $X$ refers to population data, and it is of interest to know whether similar changes could be shown to occur in each individual when the tests were repeated. The population available was smaller, but as Table XI shows, the individual's sensitivity improved for each successive test. Considering the size of the population a value of $t>3$ is very highly significant.

Evidently results in all individuals tested repeatedly are similar to those of the entire population. In years $1-2$ and $2-3$, there is a decline in threshold, more marked for tritan than protan. The mean decline for tritan is 5 and 7 times the SE of the mean, indicating a real change: for protan, the value for year 1-2 is 3 times the SE, but for years 2-3 it is only 1.5 times, so the decline is not significant.

\section{Other Sources of Variation}

Effect of Laser Use. In the previous investigation, the cumulative use of argon blue was correlated to threshold. The use, in older ophthalmologists, could have continued for many years, and therefore age is a very important variable. Therefore before further analysis the data were transformed to remove all effects of age. Although the change with age is small, it is highly significant. Thus for protan, at 20 years the

Table XI. Differences between individuals' thresholds as function of year of survey

\begin{tabular}{lccccc}
\hline & \multicolumn{2}{c}{ Test 1-2 } & & \multicolumn{2}{c}{ Test 2-3 } \\
\cline { 2 - 3 } \cline { 5 - 6 } & Protan & Tritan & & Protan & Tritan \\
\hline Number & 362 & 362 & & 125 & 125 \\
Mean difference & 0.27 & 1.01 & & 0.14 & 1.86 \\
SD & 1.60 & 3.81 & & 0.98 & 4.65 \\
SE & 0.084 & 0.200 & & 0.088 & 0.254 \\
$t$-value & -3.24 & -5.06 & & -1.59 & -3.30 \\
Significance & $0.001^{\mathrm{a}}$ & $0.000^{\mathrm{a}}$ & & 0.115 & $0.001^{\mathrm{a}}$ \\
\hline
\end{tabular}

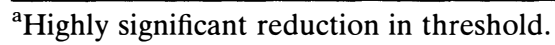


Table XII. Variables which may affect colour vision thresholds (results age- adjusted: all outliers removed from protan data)

\begin{tabular}{|c|c|c|c|c|}
\hline Variable & Constant & Slope \pm SE $(\times 1000)$ & $t \pm \mathrm{SE}$ & Sig. $(t)$ \\
\hline \multicolumn{5}{|c|}{ Protan thresholds } \\
\hline Blue argon exposure & $5.606 \pm 0.0488$ & $0.17 \pm 0.013$ & -1.515 & 0.1300 \\
\hline Green argon exposure . & $5.624 \pm 0.0508$ & $-0.12 \pm 0.1$ & -1.971 & 0.0490 \\
\hline YAG laser exposure & $5.634 \pm 0.0530$ & $-0.9 \pm 0.4$ & -2.057 & 0.0400 \\
\hline Operating microscope exposure & $5.610 \pm 0.0584$ & $-0.02 \pm 0.2$ & -0.968 & 0.3332 \\
\hline Sailing exposure & $5.594 \pm 0.0459$ & $-0.05 \pm 0.05$ & -1.108 & 0.2681 \\
\hline Skiing exposure & $5.574 \pm 0.0469$ & Tritan thresholds & -0.065 & 0.9482 \\
\hline Blue argon exposure & $11.72 \pm 0.116$ & $0.25 \pm 0.27$ & 0.94 & 0.348 \\
\hline Green argon exposure & $11.78 \pm 0.1206$ & $-0.66 \pm 0.241$ & 0.25 & 0.803 \\
\hline YAG exposure & $11.956 \pm 0.125$ & $-2.8 \pm 1.1$ & -2.61 & 0.009 \\
\hline Operating microscope exposure & $11.75 \pm 0.141$ & $0.007 \pm 0.44$ & 0.17 & 0.867 \\
\hline Sailing exposure & $11.76 \pm 0.109$ & $0.02 \pm 0.1$ & 0.22 & 0.826 \\
\hline Skiing exposure & $11.79 \pm 0.111$ & $-0.22 \pm 0.26$ & -0.83 & 0.405 \\
\hline \multicolumn{5}{|c|}{ Tritan thresholds including outliers } \\
\hline Blue argon exposure & $12.35 \pm 0.183$ & $0.1 \pm 0.4$ & 0.332 & 0.740 \\
\hline Green argon exposure & $12.50 \pm 0.190$ & $-0.5 \pm 0.4$ & -1.346 & 0.179 \\
\hline YAG exposure & $12.63 \pm 0.198$ & $-3.9 \pm 1.7$ & -2.296 & 0.022 \\
\hline Operating microscope exposure & $12.29 \pm 0.219$ & $0.04 \pm 0.07$ & 0.571 & 0.568 \\
\hline Sailing exposure & $12.41 \pm 0.172$ & $-0.11 \pm 0.18$ & 0.599 & 0.549 \\
\hline Skiing exposure & $12.38 \pm 0.175$ & $-0.06 \pm 0.41$ & -0.152 & 0.879 \\
\hline
\end{tabular}

threshold is 4.136 and at 60 it is 4.312 . The agerelated changes in threshold account for less than $1 \%$ of the variance in the population. Following this transform, it is possible to determine whether any correlation exists between the time spent on any activity (use of lasers, or skiing). These analyses were carrid out for the first tests, and are shown in Table XII.

Now, no correlation can be determined between the protan threshold and any of the other variables tested, while for tritan threshold there is no significant correlation between hours spent using laser blue light and threshold. However, in the first year of the trial the thresholds of persons who have never used argon blue lasers are significantly less than those who have ( 350 of 958), and similar to the normal values. The small number of ophthalmologists who continued to use argon blue during the first year of the survey (44) had, on average, a still higher threshold than those who had ceased to use argon blue.

Table XIII shows the thresholds after all the exclusions mentioned above and after age correction, with slightly different totals from those given in the text immediately above. However, almost all the subjects were currently employing argon green light, and the degree of continuing use of blue was very small. Thus, by the second year, only 14 were using argon blue light. Therefore the failure to establish any relation between colour contrast sensitivity and exposure to argon blue may simply reflect the fact that argon blue is no longer used, and any effect is not persistent. Because of the small numbers in the subsequent groups, the data are not reliable, but it appears that the thresholds of those who had used or were using argon blue also fell from year 1 to year 3; this may be due to increased precautions taken by the users, or a dwindling use of argon blue. Table XIII also repeats the normal values, and the mean values for ophthalmologists in the final year of the survey. It can be seen that the slight elevation of mean tritan thresholds seen in the earlier years has vanished.

Other Possible Correlations. After excluding all the outliers (who may or may not be undeclared diabetics or hypertensives) there is a slight correlation between exposure to two of the variables included in the questionnaire. However, the correlation is negative, i.e. increasing the duration of exposure to the YAG laser or argon green increases sensitivity. Such a correlation is likely to be fortuitous. Note that the values given in column 3 (the connection between duration of exposure and

Table XIII. Tritan thresholds of ophthalmologists related to use of argon blue (after excluding all persons with known diseases, or congenital colour defects, or thresholds $>2$ SDs above mean of healthy normals, age corrected)

\begin{tabular}{|c|c|c|c|c|}
\hline & $n$ & Tritan threshold & SE & SD \\
\hline Those who have never used argon blue & 592 & $11.46^{*}$ & 0.13 & 3.27 \\
\hline Those who have used argon blue & 337 & $12.24 * \dagger$ & 0.18 & 3.35 \\
\hline Those who continued to use argon blue in year 1 & 41 & $12.68 \dagger$ & 0.58 & 3.70 \\
\hline $\begin{array}{l}\text { Normal results: non laser users } \\
\text { (age adjusted to mean age of ophthalmologists-Table VII) }\end{array}$ & & $11.43 *$ & 0.032 & \\
\hline All ophthalmologists, less exclusions, year 3 (Table IV) & & 11.18 & 0.18 & \\
\hline
\end{tabular}

*Mean values significantly different: $t=2.19, \mathrm{DF}=927, p<0.05>0.02$.

$\dagger$ Mean values significantly different: $t=2.40, \mathrm{DF}=376, p=0.06$. 
threshold) are multiplied by 1000: the decrements in threshold associated with intensive use of lasers are very small indeed. Similar analyses to those of Table XII were conducted for the second and third years, with similar results. The numbers involved are smaller, and the results are not presented in detail. In addition to these tests, the entire set of data was subjected to a multiway ANOVA, and to multiple stepwise regression, ${ }^{19}$ using a computer package (STSS). No additional relationships between the variables studied were found: the variables are those listed in Table XII.

\section{DISCUSSION}

In our previous work we found elevated tritan thresholds in German ophthalmologists who used argon lasers. The limitations of the work were that all the information was all obtained in 3 days and only $20 \%$ of the population was assessed, so we did not know whether the sample was representative, and later checks were impossible. This survey was designed to provide answers which would not suffer from previous methodological defects. In two separate investigations we have shown that the results of nearly $100 \%$ of samples were the same as those in a selfselected sample, so that the general applicability of the data seems assured. In groups of normals tested through the survey, similar thresholds were obtained. Even with tests as closely spaced as 5 weeks, there is no 'learning' and the test-retest variability is small. In other respects the survey may be considered less fortunate, because it was conducted during a period in which there were considerable changes in the use of lasers and in the administration of the hospital service. The latter changes made it difficult for us to persuade ophthalmologists to be repeat tested in the second and third years.

\section{Number of Raised Thresholds among Ophthalmologists}

The number of persons who reported that they had a congenital colour defect is about half that expected: when the protan 'outliers' are added, the proportion approaches that found in previous surveys of anomalous trichromats and dichromats. The tritan thresholds are elevated in persons with systemic disease, but this may be under-reported. The worst possible interpretation of the data is that 29 of 1073 persons had impaired tritan colour vision $(2.7 \%)$, while in the previous survey ${ }^{8}$ over $30 \%$ fell into this category. However, it is likely that the true incidence of abnormal colour vision, unassociated with systemic disease, is considerably lower. Some at least of the unexplained high tritan thresholds we recorded were probably obtained from ophthalmologists who did not know, or did not care to disclose, that they had diabetes or hypertension. Thus, the major finding is that we cannot repeat our previous observation, namely that a large proportion of ophthalmologists had elevated colour contrast thresholds, to a degree which must be considered abnormal by reference to the general population. It is not possible that the previous findings were some statistical freak, and there is independent evidence that endoscopists ${ }^{20}$ and other laser technicians ${ }^{21}$ still suffer from colour vision abnormalities.

\section{Changes in Tritan Thresholds during the Survey}

However, both including and excluding these 'outliers', we find that during the course of the investigation the thresholds fell for tritan thresholds considerably more than for protan colour contrast, and by the end of the survey the protan threshold was the same as that for our normals, and the tritan threshold was not significantly different. The tritan decrease was the largest change we found between any of the groups, and is very highly significant. The only feasible explanations are that either the test we administered changed, or the subjects tested changed during the 3 years. There is no evidence that the normal results changed: besides, any change in the equipment would have been an ageing of the monitors, which should lead to an apparent decrease in sensitivity. Age is not a variable which can explain these findings, which persist after correction for the increasing age of the population. The decrease in threshold is apparent for the whole population, and is also present when individuals are repeatedly tested. The most likely explanation is that the chronic elevation of thresholds previously found is vanishing from our population, as the use of argon blue is phased out. Thus the elevations previously found are not irreversible. It is satisfactory that we have identified a minor hazard associated with the use of blue light, and have observed its disappearance with an improvement of safety measures.

We have accumulated data on a sufficient number of ophthalmologists with two systemic diseases (hypertension and diabetes) to demonstrate that elevations in colour contrast threshold occur. Since no one working as an ophthalmologist can have impaired vision, this finding adds to the growing weight of evidence that minor impairment of colour vision precedes other losses in visual function, and this has, especially for diabetics, an important implication for the role of colour vision tests in screening.

We wish to thank The George and Frederick Barclay Trust and the Department of Health for financial support, Dr Calverd for critical reading of the manuscript, all the eye departments whose co-operation made the survey possible, and $\mathrm{Mr} \mathrm{C}$. H. Hogg for technical support which made the survey successful.

Key words: Lasers, Phototoxicity, Tritanopia. 


\section{REFERENCES}

1. Diabetic Retinopathy Research Group. Photocoagulation treatment of proliferative diabetic retinopathy: second report of diabetic retinopathy study findings. Am J Ophthalmol 1978;85:82-106.

2. American National Standards Institute. American National Standard for the safe use of lasers. ANSI Z136.1, 1986.

3. Sliney D, Wolbarsht M. Safety with lasers and other optical sources. New York: Plenum Press, 1980.

4. Sliney DH, Mainster MA. Potential laser hazards to the clinician during photocoagulation. Am J Ophthalmol 1987;103:758-60.

5. Arden GB, Gunduz K, Perry S. Color vision testing with a computer graphics system. Clin Vis Sci 1988;2:303-20.

6. Gunduz K, Arden GB. Changes in colour contrast sensitivity associated with operating argon lasers. $\mathrm{Br} \mathbf{J}$ Ophthalmol 1989;73:241-6.

7. Berninger TA, Canning CR, Gunduz K, Strong N, Arden GB. Using argon laser light reduces ophthalmologists' colour contrast sensitivity. Arch Ophthalmol 1989;107:1453-8.

8. Arden GB, et al. A survey of colour discrimination in German ophthalmologists: changes associated with the use of lasers and operating microscopes. Ophthalmology 1991;98:567-75.

9. Johnson CA, Shapiro LA. A comparison of MOBS (modified binary search) and staircase test procedures in automated perimetry. OSA Tech Digest Ser 1989;7:84-7.

10. Yu TC, Falcao-Reis F, Spileers W, Arden GB. Peripheral colour contrast. Invest Ophthalmol Vis Sci 1991;32:2779-89.

11. Arden GB, Gunduz K, Perry S. Colour vision testing with a computer-graphics system: preliminary results. Doc Ophthalmol 1988;69:167-74.

12. Gunduz K, Arden GB, Perry S. A new test of colour vision using TV and computer graphics: results in some common acquired eye diseases. In: Kulikowski JJ, Dickinson CM, Murray IJ, editors. Seeing, contrast and colour. Oxford: Pergamon Press, 1988:373-9.

13. Hart WM. Acquired dyschromatopsias. Surv Ophthalmol 1987;32:10-31.

14. Scase MO, Foster DH, Honan WP, Heron JR, Guilliford MC, Scarpello JHB. Abnormalities in hue discrimination revealed with very brief stimuli in diabetes mellitus and in optic neuritis patients. Clin Vis Sci 1990;6:49-57.

15. De Alwys DV, Reffin JP, Tregear SJ, Ripley LG, Caswell AG. Should the management of diabetic retinopathy be based upon measurements of visual function rather than observations of retinal morphology? Invest Ophthalmol Vis Sci (ARVO Suppl) 1993;34:719.

16. Treagear SD, Knowles PJ, De Alwys DV, Reffin JP, Ripley LG, Caswell AG. Colour vision deficits predict the development of sight-threatening disease in diabetic subjects with background retinopathy? Invest Ophthalmol Vis Sci (ARVO Suppl) 1993;719.

17. Holz F, Bird AC, Arden GB. Longitudinal and crosssectional survey of colour vision in patients with macular drusen. German J Ophthalmol (submitted).

18. Ruddock KH. The effect of age on colour vision. I. Response in the receptoral system of the eye. Vision Res 1965;5:37-45.

19. Bryman A, Cramer D. Quantitative data analysis for social scientists. London: Routledge, 1990.

20. Koningsberger JC, van Norren D, van Neil JCG, Dekker W. Does colour vision deficiency in endoscopists influence the accuracy of endoscopic diagnosis? An anonymous study with Dutch gastrointenstinal endoscopists. Endoscopy 1994;6:549-53.

21. Arden GB, Hall M. Colour contrast thresholds in laser technicians. In preparation. 